SNUTP 07-005

\title{
PVLAS experiment, star cooling and BBN constraints: Possible interpretation with temperature dependent gauge symmetry breaking
}

\author{
Jihn E. Kim \\ Department of Physics and Astronomy and Center for Theoretical Physics, \\ Seoul National University, Seoul 151-747, Korea
}

\begin{abstract}
It is known that the kinetic mixing of photon and another $\mathrm{U}(1)_{\mathrm{ex}}$ gauge boson can introduce millicharged particles. Millicharged particles $f$ of mass $0.1 \mathrm{eV}$ can explain the PVLAS experiment. We suggest a temperature dependent gauge symmetry breaking of $\mathrm{U}(1)_{\mathrm{ex}}$ for this idea to be consistent with astrophysical and cosmological constraints.

PACS numbers: 14.80.-j, 95.30.Cq, 11.10.Wx
\end{abstract}


The possibility of dichroism of vacuum in a magnetic field has been announced by the PVLAS collaboration [1]. The vacuum magnetic dichroism arises from the absorption rate difference of two polarizations of polarized light in a magnetic field. The absorption of photons in vacuum hints that some particles light enough (milli-eV range) are produced in the apparatus. The question is what those light particles could be. One well-known possibility is an axion-like particle $(a)$ production in the magnetic field by the Primakoff interaction $\mathcal{L}_{a \gamma \gamma}=\frac{a}{8 M} \epsilon_{\mu \nu \rho \sigma} F^{\mu \nu} F^{\rho \sigma}$ where $1 \mathrm{meV} \lesssim m_{a} \lesssim 1.5 \mathrm{meV}$ and the $a$ coupling to photon in the region $2 \times 10^{5} \mathrm{GeV} \lesssim M \lesssim 6 \times 10^{5} \mathrm{GeV}$. The Primakoff process is shown in Fig. 1(a). However, the bound on $M$ is in direct contradiction with the CAST axion search result, constraining the axion decay parameter in the range $M>10^{10} \mathrm{GeV}$ [2].

This leads us to consider another light particles $f$ and $\bar{f}$ which can be produced by the photons as shown in Fig. 1(b) and $f$ is called 'millicharged' fermion [3]. For this process to be possible, we must resolve two problems, how both the $f$ mass and the photon coupling to $f$ can be so small,

$$
\begin{aligned}
& m_{f} \simeq 0.1 \mathrm{eV} \\
& \epsilon_{f} \equiv Q_{f} /|e| \simeq 3 \times 10^{-6},
\end{aligned}
$$

where $-e$ is the electron electric charge.

All observed integer electric charges of the known color singlet particles strongly suggest the quantization of the electric charges of all elementary particles. The experimental upper limits on the violation of electric charge quantization was obtained from the experiments on neutrons [4], atoms [5], and molecules [6]: $Q / e<\mathcal{O}\left(10^{-21}\right)$. If we introduce the millicharged particle $f$ with (2) in the standard model (SM), then all electromagnetic charges

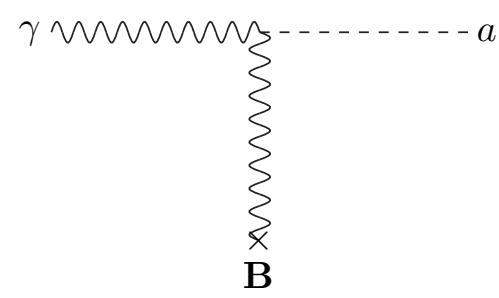

(a)

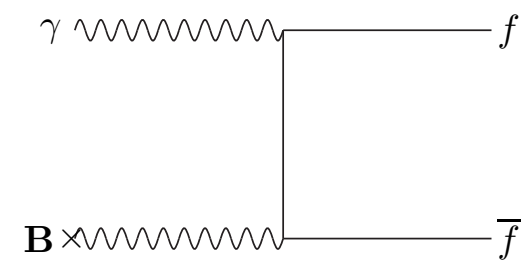

(b)

FIG. 1: Feynman diagrams leading to photon conversions in a magnetic field. 
are integer multiples of $\epsilon_{f} e$ if magnetic monopoles exist [7]. Thus a millicharged particle at the fundamental level introduces a severe problem in the SM.

But going beyond the SM, there exists a possibility of introducing such a small unquantized charge, by introducing an additional hidden-sector U(1) factor [8]. Holdom noted that the millicharge is not in conflict with the charge quantization if it gets an induced electric charge proportional to some small mixing between the kinetic terms of photons and extra (or exotic) photons [9], exphotons. For the electromagnetic $\mathrm{U}(1)_{\mathrm{em}}$ and at least one more hidden sector $\mathrm{U}(1)_{b}^{\prime}$ factor, the kinetic mixing is usually parametrized as

$$
\mathcal{L}_{\mathrm{KE}}=-\frac{1}{4} F_{(\mathrm{em})}^{\mu \nu} F_{(\mathrm{em}) \mu \nu}-\frac{1}{4} F_{(b)}^{\prime \mu \nu} F_{(b) \mu \nu}^{\prime}+\frac{\chi}{2} F_{(\mathrm{em})}^{\mu \nu} F_{(b) \mu \nu}^{\prime}
$$

where $\chi$ parametrizes the mixing. The parameter $\chi$ is directly linked to charge shifts. Eq.(3) is written in the basis where the interaction terms have the canonical form, i.e. here the electric charge quantization is manifest. For example, with two fermion species $f_{a}$ and $f_{b}$ with charges $(e, 0)$ and $(0, e)$, respectively, under $\mathrm{U}(1)_{\mathrm{em}} \times \mathrm{U}(1)_{b}^{\prime}$, after diagonalization of $\mathcal{L}_{\mathrm{KE}}$, their charges are known to be shifted by $\epsilon_{f} \simeq \chi$ in the leading order of $\chi[9]$.

However, it was argued that introducing an exphoton with millicharge (2) alone is not free from all astrophysical constraints. Stellar cores can lose energy much more efficiently by plasma production of $f$ and $\bar{f}$ compared to the energy loss via weak interactions. The current astrophysical bound on $\epsilon_{f}$ is $\epsilon \lesssim 2 \times 10^{-14}$ for $m_{f} \lesssim$ few keV [10], which is eight orders of magnitude below the one needed for the interpretation of the PVLAS data. This confrontation has led to a need for introducing at least two extra $\mathrm{U}(1)_{b}^{\prime} \mathrm{s}$ in the hidden sector for the case of Fig. 1(b) [11] and a dimension 6 coupling without the dimension 5 coupling for the case of 1(a) [12]. In particular, Masso and Redondo [11] considered an exactly massless exphoton and another light exphoton with its mass in the range, $\mathrm{keV} \gg m_{\tilde{\gamma}} \neq 0$ where $m_{\tilde{\gamma}}$ is the exphoton plasmon mass in the core of stars. The specific model considered in [11] employs a bi-fundamental representation of two extra $\mathrm{U}(1)^{\prime} \mathrm{s}, \mathrm{U}(1)_{1}^{\prime}$ and $\mathrm{U}(1)_{2}^{\prime}$, and obtain the needed condition that $\chi_{1}=\chi_{2}$ which is a fine-tuning condition. In a realistic model from superstring, however, it is not likely that the conditions as suggested in Ref. [11] are satisfied. In Fig. 2, a typical diagram contributing to the kinetic mixing between photon and $\mathrm{U}(1)_{\mathrm{ex}}^{\prime}$ gauge boson is shown. The threshold correction by heavy particles above and near the GUT scale for $\mathrm{U}(1)_{\mathrm{em}}^{\prime}$ and $\mathrm{U}(1)_{i}$ gauge boson is $\chi_{i} \simeq-\frac{e e_{i}}{16 \pi^{2}} \sum_{F} Q_{\mathrm{em}}(F) Q_{i}(F) \ln \frac{M_{F}^{2}}{\mu^{2}}$ where $e$ is the positron charge, $e_{i}$ is the $\mathrm{U}(1)_{i}$ charge, and $\mu$ is the renormalization scale. Because 


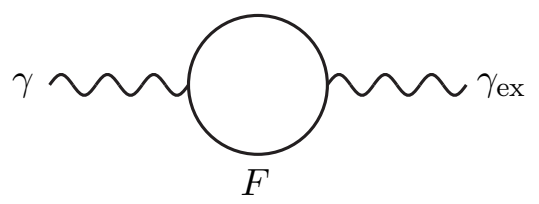

FIG. 2: Mixing of $\mathrm{U}(1)_{\mathrm{em}}$ and $\mathrm{U}(1)_{\mathrm{ex}}$ gauge bosons through a heavy particle $F$.

the heavy particle masses are not identical and $\sum_{F} Q_{\mathrm{em}}(F) Q_{1}(F) \neq \sum_{F} Q_{\mathrm{em}}(F) Q_{2}(F)$ in general, the Masso-Redondo condition is difficult to be realized.

In this paper, we introduce just one $\mathrm{U}(1)$ ex gauge symmetry and a temperature dependent $\mathrm{U}(1)_{\text {ex }}$ gauge symmetry breaking to incorporate the PVLAS data, the astrophysical and cosmological bounds. The idea is the following.

We anticipate that the PVLAS experiment has detected the kinetic mixing effect a la Fig. 1(b). So, suppose that two U(1) gauge groups survive below the electroweak scale, say $\mathrm{U}(1)_{\mathrm{em}}$ and $\mathrm{U}(1)_{\mathrm{ex}}$. The standard model fermions are neutral under $\mathrm{U}(1)_{\mathrm{ex}}$ and the fermions having nonvanishing $\mathrm{U}(1)_{\mathrm{ex}}$ charges are called exotics. There exists the mixing between $\mathrm{U}(1)_{\mathrm{em}}$ and $\mathrm{U}(1)_{\mathrm{ex}}$ as shown in Fig. 2 through exotic fermions $F$. Suppose that the mixing parameter $\chi$ turns out to be $\mathrm{O}\left(10^{-6}\right)$ to explain the PVLAS data. $\mathrm{U}(1)_{\mathrm{em}}$ and $\mathrm{U}(1)_{\mathrm{ex}}$ being good gauge symmetries below the electroweak scale, we can break one linear combination of them and still preserve the remaining combination $\tilde{\mathrm{U}}(1)_{\mathrm{em}}$ as the $\mathrm{U}(1)$ of QED. There must be a very light vectorlike exotic fermionic particle pair $f$ and $\bar{f}$ with neutral $\tilde{Q}_{\text {em }}$ charge so that their charges after diagonalization of the kinetic terms is of order $3 \times 10^{-6}$. Without confusion, we will use $f$ to represent the Dirac particle, $f$ plus $\bar{f}$. This $f$ is supposed to be the ones produced at PVLAS. But its original $Q_{\mathrm{em}}$ charge is nonzero. The spontaneous breaking of $\mathrm{U}(1)_{\mathrm{em}}$ and $\mathrm{U}(1)_{\mathrm{ex}}$ is achieved by a vacuum expectation value (VEV) of a scalar field $\phi$ at a scale somewhat below $\mathrm{keV}$. The potential of $\phi$ is such that its VEV vanishes above the critical temperature which is below $\mathrm{keV}$. Thus, at stars where $T \sim 2 \mathrm{keV}$, the $\mathrm{VEV}$ is zero and the original $\mathrm{U}(1)_{\mathrm{em}}$ is not broken. Then, in the core of a star $f$ interacts with photon with the electroweak strength and hence they are trapped in the star. Trapping of $f$ in the star is like photons' difficulty in escaping the star.

To formulate the above idea let there be a vectorlike heavy superfields $F, \bar{F}$ whose $\mathrm{U}(1)_{\mathrm{em}} \times \mathrm{U}(1)_{\text {ex }}$ charges are $\left(\frac{1}{3}, \frac{2}{3}\right)$ and $\left(-\frac{1}{3},-\frac{2}{3}\right)$. The fractional charges are used to mimick some singlet exotics of Ref. [13]. We chose the $Q_{\mathrm{em}}$ charge such that it is called exotics 
above the VEV scale of $\phi$. The charges of scalar $\phi$ are taken to be $\left(\frac{1}{3},-\frac{1}{3}\right)$. Also, the charges of $f$ and $\bar{f}$ are chosen as $\left(-\frac{1}{3}, \frac{1}{3}\right)$ and $\left(\frac{1}{3},-\frac{1}{3}\right)$, respectively. Below the critical temperature $T_{c 1} \ll \mathrm{keV}, \mathrm{U}(1)_{\mathrm{em}} \times \mathrm{U}(1)_{\mathrm{ex}}$ symmetry is broken down to $\tilde{\mathrm{U}}(1)_{\mathrm{em}}$. The $\tilde{\mathrm{U}}(1)_{\mathrm{em}}$ charges of $f$ and $\bar{f}$ are zero. Let the gauge bosons of $\mathrm{U}(1)_{\mathrm{em}}$ and $\mathrm{U}(1)_{\mathrm{ex}}$ are $A_{\mu}$ and $B_{\mu}$, respectively, whose coupling to $\phi$ is

$$
i D_{\mu} \phi \rightarrow\left(e A_{\mu} Q_{\mathrm{em}}+e_{\mathrm{ex}} B_{\mu} Q_{\mathrm{ex}}\right) \phi=\frac{1}{3}\left(e A_{\mu}-e_{\mathrm{ex}} B_{\mu}\right) \phi
$$

Thus the photon $\gamma_{\mu}$ (the gauge boson of QED) and the sub-keV exphoton $E_{\mu}$ are

$$
\gamma_{\mu}=\cos \theta A_{\mu}+\sin \theta B_{\mu}, \quad E_{\mu}=-\sin \theta A_{\mu}+\cos \theta B_{\mu}
$$

where $\tan \theta=e / e_{\mathrm{ex}}$. Because the generic value of $e / e_{\mathrm{ex}}$ is not 1 at the electroweak scale, we have generically $\cos ^{2} \theta \neq \sin ^{2} \theta$. Thus, the kinetic mixing in terms of the redefined fields is

$$
-\frac{1}{4}(1+2 \chi \sin \theta \cos \theta) \gamma_{\mu \nu} \gamma^{\mu \nu}-\frac{1}{4}(1-2 \chi \sin \theta \cos \theta) E_{\mu \nu} E^{\mu \nu}-\frac{\chi}{2}\left(\cos ^{2} \theta-\sin ^{2} \theta\right) \gamma_{\mu \nu} E^{\mu \nu} .
$$

Since $\chi$ is small, we can consider a new parameter $\tilde{\chi}=\chi\left(\cos ^{2} \theta-\sin ^{2} \theta\right)$ for the mixing parameter of $\gamma_{\mu}$ and $E_{\mu}$. It will be again of order $10^{-6}$. The particles $f$ and $\bar{f}$ have the QED charge 0 but nonvanishing $E_{\mu}$ charges $\pm \frac{1}{3} \sqrt{e^{2}+e_{\mathrm{ex}}^{2}}$. The kinetic mixing shifts the QED charge of $f$ from neutral point by the amount $\epsilon_{f}=2 \tilde{\chi} / 3 \sin \theta=\frac{2}{3} \chi(\cos \theta \cot \theta-\sin \theta)$. These are the millicharged particles for the PVLAS experiment. The millicharged particles of $\tilde{Q}_{\mathrm{em}} \sim 3 \times 10^{-6}$ are allowed from all particle physics experiments [3].

We introduce a temperature dependent potential such that at the cores of stars the gauge symmetry is restored [14]. The temperature dependence regarding Fig. 1(a) was used in Ref. [12]. Thus, at the cores of stars, the original $Q_{\mathrm{em}} \mathrm{s},-\frac{1}{3}$ and $\frac{1}{3}$, of $f$ and $\bar{f}$ are seen fully and they cannot take out the core energy efficiently. For them, it is as difficult as photons have the difficulty in escaping stars. However, they can affect the Big Bang nucleosynthesis $(\mathrm{BBN})$. The model suggested above has a few new light particles: the gauge boson $E_{\mu}$, the complex boson $\phi$, the chiral fermions $f$ and $\bar{f}$ (or the Dirac particle $f$ for short). If they are in equilibrium at BBN, their effective additional cosmologically equivalent neutrino number $\left(\delta N_{\nu}\right)$ is $\frac{8}{7}(1+1)+1+1 \simeq 4.3$ which is too large [15]. So it is necessary to break the gauge symmetry $\mathrm{U}(1)_{\mathrm{ex}}$ at the BBN temperature of order $\mathrm{MeV}$. Then, at the BBN era $f$ and $\bar{f}$ are milli-charged and they are not in equilibrium with photons and their number density can be sufficiently lowered assuming that they are decoupled at a sufficiently early time. The 
gauge boson $E_{\mu}$ is heavy at the BBN era and can be neglected. The complex boson $\phi$ has the vanishing $\tilde{Q}_{\mathrm{em}}$ charge at the $\mathrm{U}(1)_{\text {ex }}$ broken phase, and hence it is not in equilibrium with photon at the BBN era and hence its contribution to $\delta N_{\nu}$ can be neglected.

To have a successful temperature dependent symmetry breaking pattern, let us introduce the minimal setup with two more real scalars $\rho$ and $\sigma$. The mass $m$ of $\sigma$ is between $\mathrm{MeV}$ and $\mathrm{keV}$ and the mass $M$ of $\rho$ is somewhat above the MeV scale. The potential of scalar fields $\phi, \rho$, and $\sigma$ is

$$
V=-\mu^{2} \phi^{*} \phi+\frac{1}{2} m^{2} \sigma^{2}+\frac{1}{2} M^{2} \rho^{2}+\lambda_{1}\left(\phi^{*} \phi\right)^{2}-\lambda_{2} \phi^{*} \phi \sigma^{2}+\lambda_{3} \phi^{*} \phi \rho^{2}+\cdots
$$

where all parameters are taken to be positive, $\mu^{2}>0, m^{2}>0, M^{2}>0$, and $\lambda_{i}>0(i=$ $1,2,3)$, and $\cdots$ represents the quartic terms of $\rho$ and $\sigma$. The temperature dependent potential depending on $\phi$ is estimated as [14, 16, 17]

$$
V_{T}=\frac{1}{2} T^{2}\left[\lambda_{1}-\frac{1}{2} \lambda_{2} \theta(T-m)+\frac{1}{2} \lambda_{3} \theta(T-M)\right] \phi^{*} \phi
$$

where we used the unit Boltzmann constant convention, $k=1$, and $\theta(x)$ is the step function of $x$. Then, at zero temperature the gauge symmetry $\mathrm{U}(1)_{\mathrm{ex}}$ is broken by the VEV of $\phi$ : $\langle\phi\rangle=v / \sqrt{2}$, where $v=\sqrt{\mu^{2} / \lambda_{1}}$. We choose a quartic coupling hierarchy as

$$
\lambda_{1} \gg \frac{1}{2} \lambda_{2}-\lambda_{1} \gg \lambda_{1}-\frac{1}{2} \lambda_{2}+\frac{1}{2} \lambda_{3}>0
$$

Then, there exist three critical temperatures which separate broken and unbroken phases of $\mathrm{U}(1)_{\mathrm{ex}}$ :

$$
T_{c 1}=\sqrt{\frac{2 \mu^{2}}{\lambda_{1}}}, \quad T_{c 2}=\sqrt{\frac{4 \mu^{2}}{\lambda_{2}-2 \lambda_{1}}}, \quad T_{c 3}=\sqrt{\frac{4 \mu^{2}}{\lambda_{3}-\lambda_{2}+2 \lambda_{1}}}, \quad T_{c 1} \ll T_{c 2} \ll T_{c 3} .
$$

There are four phase regions

$$
\begin{aligned}
& R_{0}\left(T<T_{c 1}\right): \mathrm{U}(1)_{\mathrm{ex}} \text { broken, } \quad Q_{\mathrm{em}}(f)=\tilde{\chi} \\
& R_{1}\left(T_{c 1}<T<T_{c 2}\right): \mathrm{U}(1)_{\mathrm{ex}} \text { unbroken, } \quad Q_{\mathrm{em}}(f)=\frac{1}{3} \\
& R_{2}\left(T_{c 2}<T<T_{c 3}\right): \mathrm{U}(1)_{\mathrm{ex}} \text { broken, } \quad Q_{\mathrm{em}}(f)=\tilde{\chi} \\
& R_{3}\left(T>T_{c 3}\right): \mathrm{U}(1)_{\mathrm{ex}} \text { unbroken, } \quad Q_{\mathrm{em}}(f)=\frac{1}{3}
\end{aligned}
$$

The hierarchy of masses and critical temperatures is shown in Fig. 3. The critical temperature $T_{c 1}$ is somewhat below $1 \mathrm{keV}$ the typical temperature in astrophysical environments. The critical temperature $T_{c 2}$ is somewhat below the $\mathrm{MeV}$ scale but above a few $\mathrm{keV}$, and the 


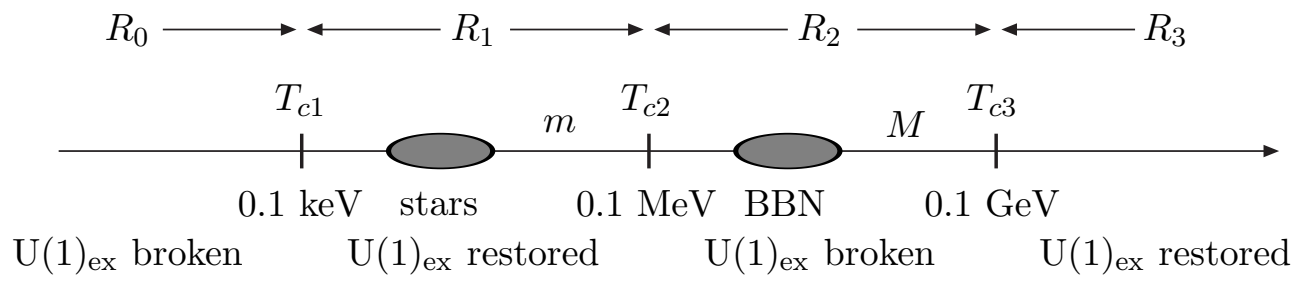

FIG. 3: Typical scales of masses and critical temperatures.

critical temperature $T_{c 3}$ is above a few $\mathrm{MeV}$ but below the electroweak scale. These critical temperatures are taken such that during the $R_{0}$ period $\mathrm{U}(1)_{\text {ex }}$ is broken with millicharged $f$, during the $R_{1}$ period $\mathrm{U}(1)_{\text {ex }}$ is restored such that $f_{\mathrm{s}}$ are in equilibrium with photons, during the $R_{2}$ period $\mathrm{U}(1)_{\mathrm{ex}}$ is broken again with milli-charged $f$, and during $R_{3}$ period $\mathrm{U}(1)_{\mathrm{ex}}$ is restored such that $f \mathrm{~s}$ are in equilibrium with photons again. $R_{0}$ contains the PVLAS region, $R_{1}$ contains the astrophysical environment and $R_{2}$ contains the $\mathrm{BBN}$ era. This hierarchy of critical temperatures given in (8) and (9) are achieved by a fine-tuning of parameters between $\lambda_{1}, \lambda_{2}$ and $\lambda_{3}$.

The spontaneous symmetry breaking of the model in Eq. (66) introduces two more real scalars, $\sigma$ and $\rho$. At the BBN era, $\rho$ is considered to be much heavier than $1 \mathrm{MeV}$ and its contribution to $\delta N_{\nu}$ can be neglected. [By the $\lambda_{3}$ coupling it is in equilibrium with $\phi$ and its number density is suppressed by the factor $e^{-M / \mathrm{MeV}}$ compared to that of $\phi$.] So at $1 \mathrm{MeV}$, we consider the real scalar $\sigma$, the real part (the Higgs boson of $\mathrm{U}(1)_{\mathrm{ex}}$ ) of the complex scalar $\phi$ and the massive gauge boson $E_{\mu}$. If their number densities are the same as the photon number density, then $\delta N_{\nu}$ would be $\frac{20}{7}$. But their masses are of order the temperature scale and we expect that there is a suppression factor of $e^{-1}$. Thus, we estimate $\delta N_{\nu} \sim 1$ which is in the allowed region [15].

Now let us show briefly how the $\mathbf{Z}_{12-I}$ model of Ref. [13] contains the needed $\mathrm{U}(1)_{\text {ex }}$ group and $f$ and $\phi$. The orbifold compactification leads to the following gauge group

$$
S U(3)_{c} \times S U(2) \times U(1)^{5} \times\left[S O(10) \times U(1)^{3}\right]^{\prime}
$$

where the electroweak hypercharge $Y$ is $Y=\left(\frac{1}{3} \frac{1}{3} \frac{1}{3} \frac{-1}{2} \frac{-1}{2} ; 000\right)\left(0^{8}\right)^{\prime}$ in the $\mathrm{E}_{8} \times \mathrm{E}_{8}^{\prime}$ space and we obtain $\sin ^{2} \theta_{W}=\frac{3}{8}$ [13]. With this hypercharge assignment, we have exotics: color exotics, doublet exotics, and singlet exotics. $f, \bar{f}$, and $\phi$ belong to singlet exotics which can be two $\eta$ s and one $\bar{\eta}$ exotics of Ref. [13]. For example, we can assign $f$ and $\bar{f}$ as $\eta_{1}$ and $\overline{\eta_{6}}$, respectively. The $\mathrm{U}(1)_{\mathrm{ex}}$ quantum number is the third entry in the hidden sector $\left(\cdot \times ; 0^{5}\right)^{\prime}$. 
All the exotics of Ref. [13] carry nonzero U(1) ex charges, and all non-exotics carry the zero $\mathrm{U}(1)_{\text {ex }}$ charge. With the above hypercharge $Y, \mathrm{U}(1)_{\text {ex }}$ gauge boson exphoton is massless. From [13], the sum of products of the charges, excluding those of $f, \bar{f}$ and $\phi$, is found as $\sum_{i} Q_{\mathrm{em}}(i) Q_{\mathrm{ex}}(i)=\frac{35}{9} e e_{\mathrm{ex}}$ where $e$ is the positron charge and $e_{\mathrm{ex}}$ is the unit charge of exotics.

To estimate $\chi$, let us consider a toy model with two chiral superfields of charges $\left(Q_{a}, Q_{b}\right)$ and $\left(Q_{a},-Q_{b}\right)$ and masses $m$ and $m^{\prime}$ respectively, their joint contribution to $\chi$ has the form [18],

$$
\chi=-\frac{g_{a} g_{b}}{16 \pi^{2}} Q_{a} Q_{b} \log \left(\frac{m^{2}}{m^{\prime 2}}\right) .
$$

From the exotics of Ref. [13], the contribution to $\chi$ is not negligible. On general grounds, its

size is estimated as [18], $10^{-3}<\chi<10^{-2}$, where $\alpha_{Y}\left(M_{\mathrm{GUT}}\right) \sim \frac{1}{60}-\frac{1}{25}$ is used. It is possible to reduce $\chi$ to $O\left(10^{-6}\right)$ by mass parameters of the exotics and the RG running. This kind of reduction is necessary even if we obtained $\sum_{i} Q_{\mathrm{em}}(i) Q_{\mathrm{ex}}(i)=0$ due to the appearance of logarithms in Eq. (12). Thus, to derive the millicharged particle scenario, we need two kinds of fine tunings, one in the quartic coupling constants $\lambda_{1}, \lambda_{2}, \lambda_{3}$ and the other in the mass parameters of heavy exotic particles.

In conclusion, the millicharged particles whose properties have nontrivial temperature dependence can explain the PVLAS data which was unexpected from conventional models. As shown here and in other references [11, 12], it is very unnatural if not impossible to explain the PVLAS data with field theory models. The conclusions of these unnatural models, however, lead to far-reaching consequences on their origins and a crucial clue to new physics beyond the SM might be obtained by confirming its validity.

\section{Acknowledgments}

I thank K. Dienes, I.-W. Kim and B. Kyae for correspondences and numerous discussions. This work is supported in part by the KRF ABRL Grant No. R14-2003-012-01001-0. J.E.K. is also supported in part by the KRF grants, No. R02-2004-000-10149-0 and No. KRF-2005084-C00001.

[1] E. Zavattini et al. [PVLAS Collaboration], Phys. Rev. Lett. 96 (2006) 110406.

[2] K. Zioutas et al. [CAST Collaboration], Phys. Rev. Lett. 94 (2005) 121301. 
[3] H. Gies, J. Jaeckel and A. Ringwald, Phys. Rev. Lett. 97 (402) 140.

[4] M. Marinelli and G. Morpurgo, Phys. Lett. B137 (1984) 439.

[5] H. F. Dylla and J. G. King, Phys. Rev. A 7, 1224 (1973).

[6] J. Baumann, J. Kalus, R. Gahler and W. Mampe, Phys. Rev. D37 (1988) 3107.

[7] P. A. M. Dirac, Proc. Roy. Soc. Lond. A 133, 60 (1931).

[8] L. B. Okun, Sov. Phys. JETP 56, 502 (1982).

[9] B. Holdom, Phys. Lett. B166 (1986) 196, ibid. 178, 65 (1986); L. B. Okun, Sov. Phys. JETP 56, 502 (1982) [Zh. Eksp. Teor. Fiz. 83, 892 (1982)]. In the literature 'paraphoton' is used, but here we will use exphoton throughout the paper because in our model it couples to exotic particles only.

[10] S. Davidson, B. Campbell and D. Bailey, Phys. Rev. D43 (1991) 2314; S. Davidson and M. Peskin, Phys. Rev. D49 (1994) 2114; S. Davidson, S. Hannestad and G. Raffelt, JHEP 05 (2000) 003.

[11] E. Masso and J. Redondo, Phys. Rev. Lett. 97 (1996) 151802.

[12] R. N. Mohapatra and S. Nasri, Phys. Rev. Lett. 98 (2007) 050402.

[13] J. E. Kim, J.-H. Kim and B. Kyae, "Superstring standard model from $\mathbf{Z}_{12-I}$ orbifold compactification with and without exotics, and R-parity", hep-ph/0702278.

[14] D. A. Kirzhnits and A. Linde, Phys. Lett. B42 (1972) 471; L. Dolan and R. Jackiv, Phys. Rev. D9 (1974) 3320; S. Weinberg, Phys. Rev. D9 (1974) 3357.

[15] R. H. Cyburt, B. D. Fields, K. A. Olive, and E. Skillman, Astropart. Phys. 23 (2005) 313.

[16] R. N. Mohapatra and G. Senjanovic, Phys. Rev. Lett. 42 (1979) 1651.

[17] We neglected the Yukawa couplings.

[18] K. R. Dienes, C. F. Kolda and J. March-Russell, Nucl. Phys. B492 (1997) 104. 